\title{
THE REMAINDER TERM FOR ANALYTIC FUNCTIONS OF SYMMETRIC GAUSSIAN QUADRATURES
}

\author{
THOMAS SCHIRA
}

\begin{abstract}
For analytic functions the remainder term of Gaussian quadrature rules can be expressed as a contour integral with kernel $K_{n}$. In this paper the kernel is studied on elliptic contours for a great variety of symmetric weight functions including especially Gegenbauer weight functions. First a new series representation of the kernel is developed and analyzed. Then the location of the maximum modulus of the kernel on suitable ellipses is determined. Depending on the weight function the maximum modulus is attained at the intersection point of the ellipse with either the real or imaginary axis. Finally, a detailed discussion for some special weight functions is given.
\end{abstract}

\section{INTRODUCTION}

Consider the $n$-point Gaussian quadrature rule with respect to some nonnegative and integrable weight function $w$ on the interval $(-1,1)$,

$$
\int_{-1}^{1} f(x) w(x) d x=\sum_{\nu=1}^{n} w_{\nu}^{(n)} f\left(x_{\nu}^{(n)}\right)+R_{n}(f)
$$

where the knots $x_{1}^{(n)}, \ldots, x_{n}^{(n)}$ are the zeros of the $n$ th-degree orthogonal polynomial $\pi_{n}$ associated with $w$, and $w_{\nu}^{(n)}, \nu=1, \ldots, n$, are the corresponding weights (cf. [2]).

For integrands $f$ having an analytic extension into a domain $G$ (containing $[-1,1])$ it is well known that the remainder term $R_{n}(f)$ can be expressed as a contour integral. The most common contours are concentric circles or confocal ellipses. In this paper we are concerned with confocal ellipses (having foci at \pm 1 , sum of semiaxis equal to $\varrho$ and length $L\left(\mathcal{E}_{\varrho}\right)$ ),

$$
\mathcal{E}_{\varrho}:=\left\{z \in \mathbb{C}: z=\frac{1}{2}\left(\varrho e^{i \theta}+\varrho^{-1} e^{-i \theta}\right), 0 \leq \theta \leq 2 \pi\right\}, \quad \varrho>1 .
$$

Since $\mathcal{E}_{\varrho}$ shrinks to the interval $[-1,1]$ as $\varrho \searrow 1$, there exists a maximal parameter $\varrho_{\max }$ such that $f$ is analytic inside $\mathcal{E}_{\varrho}$ for $1<\varrho<\varrho_{\max }$. The contour integral representation then reads

$$
R_{n}(f)=\frac{1}{2 \pi i} \int_{\mathcal{E}_{\varrho}} K_{n}(z) f(z) d z, \quad 1<\varrho<\varrho_{\max }
$$

Received by the editor February 12, 1995 and, in revised form, January 26, 1996

1991 Mathematics Subject Classification. Primary 41A55; Secondary 65D30, 65D32.

Key words and phrases. Gaussian quadrature, remainder term for analytic functions, contour integral representation, kernel function.

(C) 1997 American Mathematical Society 
where the kernel $K_{n}$ is given by

$$
K_{n}(z):=R_{n}\left(\frac{1}{z-\cdot}\right)=\int_{-1}^{1} \frac{w(x)}{z-x} d x-\sum_{\nu=1}^{n} \frac{w_{\nu}^{(n)}}{z-x_{\nu}^{(n)}},
$$

or, alternatively, by (cf. [7])

$$
K_{n}(z)=\frac{\varrho_{n}(z)}{\pi_{n}(z)}, \quad \varrho_{n}(z):=\int_{-1}^{1} \frac{\pi_{n}(x)}{z-x} w(x) d x, z \in \mathbb{C} \backslash[-1,1] .
$$

From (1.2) one obtains the error bound

$$
\left|R_{n}(f)\right| \leq \inf _{1<\varrho<\varrho_{\max }}\left(\frac{L\left(\mathcal{E}_{\varrho}\right)}{2 \pi} \max _{z \in \mathcal{E}_{\varrho}}\left|K_{n}(z)\right| \max _{z \in \mathcal{E}_{\varrho}}|f(z)|\right) .
$$

The knowledge of the maximum modulus of the kernel $K_{n}$ is essential to obtain sharp error bounds. There is an extensive literature studying (1.5); in most articles $\left|K_{n}(z)\right|, z \in \mathcal{E}_{\varrho}$, is bounded from above or estimated asymptotically for large values of $n$ or $\varrho$ (see $[2,5,7]$ for references). W. Gautschi and R.S. Varga (cf. [7]) considered the four Chebyshev weights $w(x):=(1-x)^{\alpha}(1+x)^{\beta},|\alpha|=|\beta|=\frac{1}{2}$, and determined the point where the corresponding kernel attains its maximum modulus on $\mathcal{E}_{\varrho}$. For these kernels there exist explicit expressions based on the well-known relations

$$
T_{n}(z)=\frac{1}{2}\left(u^{n}+u^{-n}\right) \quad \text { and } \quad U_{n}(z)=\frac{u^{n+1}-u^{-n-1}}{u-u^{-1}}, \quad z=\frac{1}{2}\left(u+u^{-1}\right),
$$

for the Chebyshev polynomials of the first and second kind. By means of polar coordinates $u=\varrho e^{i \theta}, \theta \in[0,2 \pi)$, their results follow after some elementary but rather tricky calculations (cf. $[7,8]$ ). Some of the results are extended to GaussRadau and Gauss-Lobatto quadrature (cf. [4, 5, 6]). For general weight functions such explicit formulae are not known, and the question of locating the maximum modulus of $K_{n}(z)$ on $\mathcal{E}_{\varrho}$ is still unsolved in the literature.

In this paper we study the kernel of Gaussian quadrature rules with respect to symmetric weight functions $w$, for which $w(x) \sqrt{1-x^{2}}$ is increasing on $(0,1)$ or $w(x) / \sqrt{1-x^{2}}$ is decreasing on $(0,1)$. For example, the Gegenbauer weight functions $w^{(\alpha)}(x):=\left(1-x^{2}\right)^{\alpha},|\alpha|>1 / 2$, satisfy these properties. We show that on suitable ellipses $\mathcal{E}_{\varrho}, \varrho \geq \varrho_{n}^{*}$, the kernel $K_{n}$ attains its maximum modulus for $z=\frac{1}{2}\left(\varrho+\varrho^{-1}\right)$ in the first case, and for $z=\frac{i}{2}\left(\varrho-\varrho^{-1}\right)$ in the second case. The parameters $\varrho_{n}^{*}$ are explicitly given and are about 2 or $1+\sqrt{2}$, respectively (see Theorem 3.2). The error bound (1.5) is practically available since there exists a very effective algorithm for the pointwise calculation of $K_{n}(z)$ based on (1.4) and the three-term recurrence relation of the corresponding orthogonal polynomials (cf. [7]). The key of the results is a new series representation of the kernel (see Theorem 3.1), by which it is sufficient to maximize the terms of this series (see Lemma 3.1). With the help of the Chebyshev polynomials of the first and second kind the maximization of the terms is split into smaller and explicitly solvable maximization problems (see Lemmas $4.2-4.4$ ). Combining these lemmas, we are led to a short proof of the main result presented in Theorem 3.2. The paper ends with a discussion of some special weight functions. 


\section{Preliminary Lemmas}

In the sequel we consider Gaussian quadrature rules with respect to symmetric weight functions $w$ on $(-1,1)$, i.e., $w(-x)=w(x), x \in(-1,1)$. We denote by $\pi_{n}$ the $n$ th-degree orthonormal polynomial with leading coefficient $c_{n}>0$ and zeros $x_{k}^{(n)}, k=1, \ldots, n$, arranged in decreasing order $-1<x_{n}^{(n)}<\cdots<x_{1}^{(n)}<1$. The zeros are symmetric, i.e., $x_{n+1-k}^{(n)}=-x_{k}^{(n)}, k=1, \ldots, n$, and hence

$$
\pi_{n}(x)=c_{n} x^{n-2\left[\frac{n}{2}\right]} \prod_{k=1}^{\left[\frac{n}{2}\right]}\left(x^{2}-\left(x_{k}^{(n)}\right)^{2}\right) .
$$

A well-known inequality between the zeros of orthogonal polynomials corresponding to different weights $w$ is needed, for which we present an elementary proof based on [1, Satz 48].

Lemma 2.1. Let $w$ and $\widetilde{w}$ be two symmetric weight functions on $(-1,1)$ and let the zeros $x_{k}^{(n)}$ and $\widetilde{x}_{k}^{(n)}, k=1, \ldots, n$, of the corresponding $n$ th-degree orthogonal polynomials $\pi_{n}$ and $\widetilde{\pi}_{n}$, respectively, be arranged in decreasing order. If $w / \widetilde{w}$ is increasing on $(0,1)$ (in particular, $\widetilde{w}>0$ ), then the inequalities $x_{k}^{(n)} \geq \widetilde{x}_{k}^{(n)}$ hold for $k=1, \ldots,\left[\frac{n}{2}\right]$.

Proof. Assume $w / \widetilde{w}$ to be nonconstant on $(0,1)$, since otherwise $\pi_{n}$ and $\widetilde{\pi}_{n}$ have the same zeros. For arbitrary but fixed $k \in\left\{1, \ldots,\left[\frac{n}{2}\right]\right\}$ set $\lambda_{k}:=w\left(\widetilde{x}_{k}^{(n)}\right) / \widetilde{w}\left(\widetilde{x}_{k}^{(n)}\right) \geq 0$ and construct the $(2 n-2)$ nd-degree polynomial

$$
p(x):=\prod_{\nu=1}^{k-1}\left(x^{2}-\left(x_{\nu}^{(n)}\right)^{2}\right)^{2}\left(x^{2}-\left(\widetilde{x}_{k}^{(n)}\right)^{2}\right) \prod_{\nu=k+1}^{\left[\frac{n}{2}\right]}\left(x^{2}-\left(\widetilde{x}_{\nu}^{(n)}\right)^{2}\right)^{2} x^{2\left(n-2\left[\frac{n}{2}\right]\right)} .
$$

Obviously, $p(x) \leq 0$, if $|x| \leq \widetilde{x}_{k}^{(n)}$, and $p(x) \geq 0$, if $|x| \geq \widetilde{x}_{k}^{(n)}$. Because of symmetry of $w$ and $\widetilde{w}$ and monotonicity of $w / \widetilde{w}$ on $(0,1)$ the function $w(x)-\lambda_{k} \widetilde{w}(x)$ is also nonpositive for $|x| \leq \widetilde{x}_{k}^{(n)}$ and nonnegative for $|x| \geq \widetilde{x}_{k}^{(n)}$. Therefore, one has $p(x)\left(w(x)-\lambda_{k} \widetilde{w}(x)\right) \geq 0, x \in(-1,1)$, and

$$
0<\int_{-1}^{1} p(x)\left(w(x)-\lambda_{k} \widetilde{w}(x)\right) d x=\sum_{\nu=1}^{n} w_{\nu}^{(n)} p\left(x_{\nu}^{(n)}\right)-\lambda_{k} \sum_{\nu=1}^{n} \widetilde{w}_{\nu}^{(n)} p\left(\widetilde{x}_{\nu}^{(n)}\right),
$$

since the polynomial $p$ of degree $2 n-2$ is integrated exactly by the Gaussian quadrature rules relative to $w$ and $\widetilde{w}$ (with knots $x_{\nu}^{(n)}$ and $\widetilde{x}_{\nu}^{(n)}$ and positive weights $w_{\nu}^{(n)}$ and $\widetilde{w}_{\nu}^{(n)}$, respectively). The construction of $p$ and the symmetry of the zeros imply

$$
0<\sum_{\nu=k}^{\left[\frac{n}{2}\right]} w_{\nu}^{(n)} p\left(x_{\nu}^{(n)}\right)-\lambda_{k} \sum_{\nu=1}^{k-1} \widetilde{w}_{\nu}^{(n)} p\left(\widetilde{x}_{\nu}^{(n)}\right) \leq \sum_{\nu=k}^{\left[\frac{n}{2}\right]} w_{\nu}^{(n)} p\left(x_{\nu}^{(n)}\right) .
$$

Hence, there exists an index $\nu_{0} \in\left\{k, \ldots,\left[\frac{n}{2}\right]\right\}$ with $p\left(x_{\nu_{0}}^{(n)}\right)>0$, i.e., $x_{\nu_{0}}^{(n)} \geq \widetilde{x}_{k}^{(n)}$. The decreasing order of the zeros finally shows that $x_{k}^{(n)} \geq x_{\nu_{0}}^{(n)} \geq \widetilde{x}_{k}^{(n)}$.

Remark 2.1. For $\widetilde{w}>0$ on $(0,1)$ and $w / \widetilde{w}$ decreasing on $(0,1)$ there holds $x_{k}^{(n)} \leq$ $\widetilde{x}_{k}^{(n)}, k=1, \ldots,\left[\frac{n}{2}\right]$. This is proved analogously by reversing the roles of $x_{k}^{(n)}$ and $\widetilde{x}_{k}^{(n)}, k=1, \ldots,\left[\frac{n}{2}\right]$. 
Let $m \in \mathbb{N}$ and $\varrho>1$. The auxiliary quantities (cf. $[5,6,7]$ )

$$
a_{m}(\varrho):=\frac{1}{2}\left(\varrho^{m}+\varrho^{-m}\right) \quad \text { and } \quad b_{m}(\varrho):=\frac{1}{2}\left(\varrho^{m}-\varrho^{-m}\right)
$$

satisfy some elementary but useful properties.

Lemma 2.2. For $a_{m}(\varrho)$ and $b_{m}(\varrho), m \in \mathbb{N}$, there holds:

(a) $b_{m}(\varrho) \geq m b_{1}(\varrho)$ for any $\varrho>1$,

(b) $\frac{1}{m+1} b_{m+1}(\varrho)>\frac{1}{m} b_{m}(\varrho)$ for any $\varrho>1$,

(c) $\frac{1}{m+1} a_{m+1}(\varrho)>\frac{1}{m} a_{m}(\varrho)$ for any $\varrho>\frac{1}{2}(1+\sqrt{3}+\sqrt[4]{12})$.

(d) Let $1 \leq k<m$ be fixed; then the quotient $a_{m}(\varrho) / a_{k}(\varrho)$ is strictly increasing for $\varrho>1$, and $a_{m}(\varrho) / b_{k}(\varrho)$ is strictly decreasing for $\varrho>\frac{1}{2}(1+\sqrt{3}+\sqrt[4]{12})$.

Proof. (a) is equivalent to the first inequality in [6, Lemma 3.2], since $a_{m}^{2}(\varrho)=$ $b_{m}^{2}(\varrho)+1$.

(b) is valid for $m \geq 1$ and $\varrho>1$, since $\lim _{\varrho \rightarrow 1+}\left(\frac{1}{m+1} b_{m+1}(\varrho)-\frac{1}{m} b_{m}(\varrho)\right)=0$ and

$$
\begin{aligned}
2 \frac{d}{d \varrho}\left(\frac{b_{m+1}(\varrho)}{m+1}-\frac{b_{m}(\varrho)}{m}\right) & =\varrho^{m}+\varrho^{-m-2}-\varrho^{m-1}-\varrho^{-m-1} \\
& =\left(\varrho^{m-1}-\varrho^{-m-2}\right)(\varrho-1)>0 .
\end{aligned}
$$

(c) An elementary calculation shows for $m \geq 1$

$$
\frac{m}{m+1} \frac{a_{m+1}(\varrho)}{a_{m}(\varrho)}=\frac{m}{m+1} \frac{\varrho^{m+1}+\varrho^{-m-1}}{\varrho^{m}+\varrho^{-m}} \geq \frac{1}{2} \frac{\varrho^{2}+\varrho^{-2}}{\varrho+\varrho^{-1}}=\frac{a_{2}(\varrho)}{2 a_{1}(\varrho)} .
$$

The monotonicity property now holds for all $m \geq 1$ if $\varrho^{2}+\varrho^{-2}-2\left(\varrho+\varrho^{-1}\right)>0$, i.e., $\varrho+\varrho^{-1}>1+\sqrt{3}$, or equivalently $\varrho>\varrho^{*}:=\frac{1}{2}(1+\sqrt{3}+\sqrt[4]{12})$.

(d) For $1 \leq k<m$ and $\varepsilon= \pm 1$ one obtains

$$
\frac{d}{d \varrho}\left(\frac{\varrho^{m}+\varrho^{-m}}{\varrho^{k}+\varepsilon \varrho^{-k}}\right)=\frac{(m-k)\left(\varrho^{m+k}-\varepsilon \varrho^{-m-k}\right)+(m+k) \varepsilon\left(\varrho^{m-k}-\varepsilon \varrho^{-m+k}\right)}{\varrho\left(\varrho^{k}+\varepsilon \varrho^{-k}\right)^{2}} .
$$

In the case $\varepsilon=1$, this obviously implies $\frac{d}{d \varrho}\left(\frac{a_{m}(\varrho)}{a_{k}(\varrho)}\right)>0$ for $\varrho>1$, whereas in the case $\varepsilon=-1$, Lemma $2.2 \mathrm{c}$ is used to obtain $\frac{d}{d \varrho}\left(\frac{a_{m}(\varrho)}{b_{k}(\varrho)}\right)>0$ for $\varrho>\varrho^{*}$. Thus the monotonicity properties follow.

\section{The maximum modulus of the KERNEL ON Elliptic CONTOURS}

As mentioned at the beginning, the key point for determining the maximum modulus $\left|K_{n}(z)\right|$ on $\mathcal{E}_{\varrho}$ is an appropriate series representation of the kernel. In this section we develop and discuss this representation. We complete the section with formulating the results concerning the location of the maximum point of $\left|K_{n}(z)\right|$ on $\mathcal{E}_{\varrho}$.

Theorem 3.1. The kernel $K_{n}$ of a Gaussian quadrature rule with respect to a symmetric weight function $w$ on $(-1,1)$ satisfies

$$
K_{n}(z)=\sum_{\nu=0}^{\infty} \frac{c_{n+2 \nu+2}}{c_{n+2 \nu}} \frac{z}{\pi_{n+2 \nu}(z) \pi_{n+2 \nu+2}(z)} \quad(z \in \mathbb{C} \backslash[-1,1]),
$$

where $c_{k}$ is the leading coefficient of the orthonormal polynomial $\pi_{k}(c f .(2.1))$. 
Proof. According to (1.4) we have for $n \in \mathbb{N}$

$$
K_{n}(z)-K_{n+2}(z)=\frac{1}{\pi_{n}(z) \pi_{n+2}(z)} \int_{-1}^{1} \frac{\pi_{n+2}(z) \pi_{n}(x)-\pi_{n+2}(x) \pi_{n}(z)}{z-x} w(x) d x .
$$

The three-term recurrence relation of the orthonormal polynomials,

$$
\pi_{\nu+1}(x)=\frac{c_{\nu+1}}{c_{\nu}} x \pi_{\nu}(x)-\frac{c_{\nu+1} c_{\nu-1}}{c_{\nu}^{2}} \pi_{\nu-1}(x), \quad \nu=1,2, \ldots,
$$

and the formula of Christoffel-Darboux (cf. [12]) yield

$$
\begin{aligned}
& \pi_{n+2}(z) \pi_{n}(x)-\pi_{n+2}(x) \pi_{n}(z) \\
& =\frac{c_{n+2}}{c_{n+1}}\left((z-x) \pi_{n+1}(x) \pi_{n}(z)+z\left(\pi_{n+1}(z) \pi_{n}(x)-\pi_{n+1}(x) \pi_{n}(z)\right)\right) \\
& =(z-x)\left(\frac{c_{n+2}}{c_{n+1}} \pi_{n+1}(x) \pi_{n}(z)+z \frac{c_{n+2}}{c_{n}} \sum_{\nu=0}^{n} \pi_{\nu}(z) \pi_{\nu}(x)\right) .
\end{aligned}
$$

Inserting this into (3.2) and integrating we obtain, by orthonormality of the $\pi_{k}$,

$$
K_{n}(z)-K_{n+2}(z)=\frac{c_{n+2}}{c_{n}} \frac{z}{\pi_{n}(z) \pi_{n+2}(z)} .
$$

Hence, for $m \geq 1$,

$$
\begin{aligned}
K_{n}(z)-K_{n+2 m}(z) & =\sum_{\nu=0}^{m-1}\left(K_{n+2 \nu}(z)-K_{n+2 \nu+2}(z)\right) \\
& =\sum_{\nu=0}^{m-1} \frac{c_{n+2 \nu+2}}{c_{n+2 \nu}} \frac{z}{\pi_{n+2 \nu}(z) \pi_{n+2 \nu+2}(z)} .
\end{aligned}
$$

One has $\lim _{m \rightarrow \infty} K_{n+2 m}(z)=\lim _{m \rightarrow \infty} R_{n+2 m}\left(f_{z}\right)=0$ since $f_{z}(x):=(z-x)^{-1}$ is continuous for $x \in[-1,1]$ and $z \notin[-1,1]$. Thus, taking $m \rightarrow \infty$, we obtain (3.1).

Remark 3.1. Note that for symmetric weight functions the series (3.1) is a variant of Freud's formula (cf. [2, p. 308], [3])

$$
K_{n}(z)=\sum_{\nu=n}^{\infty} \frac{c_{\nu+1}}{c_{\nu}} \frac{1}{\pi_{\nu}(z) \pi_{\nu+1}(z)} .
$$

The terms in the expansion (3.1) are of order $\mathcal{O}\left(z^{-(2 n+4 \nu+1)}\right)$ for $|z| \rightarrow \infty$, i.e., they tend to zero very rapidly. A rather good approximation to $K_{n}(z)$ is obtained by taking only a few terms of (3.1) instead of the whole series. But most important for our purposes is the structure of the expansion (3.1). It reduces the determination of the maximum modulus of $K_{n}$ on $\mathcal{E}_{\varrho}$ to studying the terms of the series.

Lemma 3.1. Let $\varrho>1$ and $\psi_{\nu}(z):=\frac{z}{\pi_{n+2 \nu}(z) \pi_{n+2 \nu+2}(z)}, n \in \mathbb{N}, \nu \geq 0$. For fixed $z_{0} \in\left\{\frac{1}{2}\left(\varrho+\varrho^{-1}\right), \frac{i}{2}\left(\varrho-\varrho^{-1}\right)\right\}$ the following is valid:

$$
\text { If } \max _{z \in \mathcal{E}_{\varrho}}\left|\psi_{\nu}(z)\right|=\left|\psi_{\nu}\left(z_{0}\right)\right| \text { for all } \nu \geq 0 \text {, then } \max _{z \in \mathcal{E}_{\varrho}}\left|K_{n}(z)\right|=\left|K_{n}\left(z_{0}\right)\right| \text {. }
$$

Proof. If $z_{0}=\frac{1}{2}\left(\varrho+\varrho^{-1}\right)$, equation $(2.1)$ shows that $\pi_{m}\left(\frac{1}{2}\left(\varrho+\varrho^{-1}\right)\right)>0$ and therefore $\left|\psi_{\nu}\left(z_{0}\right)\right|=\psi_{\nu}\left(\frac{1}{2}\left(\varrho+\varrho^{-1}\right)\right)>0$ for $\nu \geq 0$. 
If $z_{0}=\frac{i}{2}\left(\varrho-\varrho^{-1}\right)$, then $(2.1)$ shows $\left|\pi_{m}\left(z_{0}\right)\right|=(-1)^{\left[\frac{m}{2}\right]} i^{2\left[\frac{m}{2}\right]-m} \pi_{m}\left(z_{0}\right)$ and therefore $\left|\psi_{\nu}\left(z_{0}\right)\right|=i(-1)^{n} \psi_{\nu}\left(\frac{i}{2}\left(\varrho-\varrho^{-1}\right)\right)$ for $\nu \geq 0$.

In both cases there exists $\vartheta \in[0,2 \pi)$, depending on $z_{0}$ and $n$ but not on $\nu$, such that $\left|\psi_{\nu}\left(z_{0}\right)\right|=e^{i \vartheta} \psi_{\nu}\left(z_{0}\right)$ for all $\nu \geq 0$. Hence, it follows from (3.1) with fixed $z_{0} \in\left\{\frac{1}{2}\left(\varrho+\varrho^{-1}\right), \frac{i}{2}\left(\varrho-\varrho^{-1}\right)\right\}$ that

$$
\max _{z \in \mathcal{E}_{\varrho}}\left|K_{n}(z)\right| \leq \sum_{\nu=0}^{\infty} \frac{c_{n+2 \nu+2}}{c_{n+2 \nu}} \max _{z \in \mathcal{E}_{\varrho}}\left|\psi_{\nu}(z)\right|=\sum_{\nu=0}^{\infty} \frac{c_{n+2 \nu+2}}{c_{n+2 \nu}}\left|\psi_{\nu}\left(z_{0}\right)\right|=e^{i \vartheta} K_{n}\left(z_{0}\right),
$$

i.e., $K_{n}(z)$ attains its maximum modulus at $z_{0} \in \mathcal{E}_{\varrho}$.

Theorem 3.1 in connection with Lemma 3.1 is the key for locating the maximum point of $\left|K_{n}(z)\right|$ on $\mathcal{E}_{\varrho}$ for Gaussian quadrature rules with respect to symmetric weight functions $w$ on $(-1,1)$, for which $w(x) \sqrt{1-x^{2}}$ is increasing on $(0,1)$ or $w(x) / \sqrt{1-x^{2}}$ is decreasing on $(0,1)$. The main result is presented in Theorem 3.2, whose proof is given in the next section.

Theorem 3.2. The kernel $K_{n}$ of a Gaussian quadrature rule with respect to a symmetric weight function $w$ on $(-1,1)$ satisfies

(a) if $w(x) \sqrt{1-x^{2}}$ is increasing on $(0,1)$, then

$$
\max _{z \in \mathcal{E}_{\varrho}}\left|K_{n}(z)\right|=K_{n}\left(\frac{1}{2}\left(\varrho+\varrho^{-1}\right)\right) \quad \text { for } \quad \varrho \geq \varrho_{n}^{*}:= \begin{cases}2.4139, & n=2, \\ 2.0017, & n=3, \\ \frac{\sqrt{2}}{2}(1+\sqrt{3}), & n \geq 4\end{cases}
$$

(b) if $\frac{w(x)}{\sqrt{1-x^{2}}}$ is decreasing on $(0,1)$, then

$$
\max _{z \in \mathcal{E}_{\varrho}}\left|K_{n}(z)\right|=\left|K_{n}\left(\frac{i}{2}\left(\varrho-\varrho^{-1}\right)\right)\right| \quad \text { for } \varrho \geq \varrho_{n}^{*},
$$

where $\varrho_{n}^{*}:=1+\sqrt{2}$ if $n \geq 1$ is odd, and if $n \geq 2$ is even, $\varrho_{n}^{*}$ is the greatest zero of

$$
d_{n}(\varrho):=\left(\varrho-\varrho^{-1}\right)^{2}-4-\left(\varrho^{2}-\varrho^{-2}\right)^{2}\left(\frac{(n+1)^{2}}{\left(\varrho^{n+1}+\varrho^{-n-1}\right)^{2}}+\frac{(n+3)^{2}}{\left(\varrho^{n+3}+\varrho^{-n-3}\right)^{2}}\right) .
$$

In Table 1 the parameters $\varrho_{n}^{*}$ of Theorem $3.2 \mathrm{~b}$ are displayed for several values of $n$. They are calculated with Newton's method and rounded towards the last given digit. Obviously, $\varrho_{n}^{*}$ converges rapidly towards $1+\sqrt{2}$ with increasing $n$.

TABLE 1

\begin{tabular}{c|c||c|c}
$n$ & $\varrho_{n}^{*}$ & $n$ & $\varrho_{n}^{*}$ \\
\hline 2 & 2.670603007 & 12 & 2.414213696 \\
4 & 2.439298097 & 14 & 2.414213568 \\
6 & 2.415739045 & 16 & 2.414213563 \\
8 & 2.414287922 & 20 & 2.41421356237 \\
10 & 2.414216825 & 100 & 2.414213562373184 \\
\hline
\end{tabular}




\section{Proof of Theorem 3.2}

The proof of Theorem 3.2 rests on Theorem 3.1 and a study of the terms $\psi_{\nu}$ (cf. Lemma 3.1). In terms of the Chebyshev polynomials of the first and second kind, the problem reduces to the maximization of the quotients $\frac{T_{n}}{\pi_{n}}$ and $\frac{z}{T_{n}(z) T_{n+2}(z)}$ on $\mathcal{E}_{\varrho}$, and of $\frac{U_{n}}{\pi_{n}}$ and $\frac{z}{U_{n}(z) U_{n+2}(z)}$ on $\mathcal{E}_{\varrho}$, respectively. We first investigate these quotients and then combine the respective results of Lemmas $4.1-4.4$ to get a short proof of Theorem 3.2.

Lemma 4.1. The function $g(z):=\frac{z^{2}-s^{2}}{z^{2}-t^{2}}$ with $s, t \in(0,1)$ has the property that on every ellipse $\mathcal{E}_{\varrho}$ with $\varrho \geq \varrho^{*}:=\frac{\sqrt{2}}{2}(1+\sqrt{3})$ there holds

$$
\max _{z \in \mathcal{E}_{\varrho}}|g(z)|= \begin{cases}g\left(\frac{1}{2}\left(\varrho+\varrho^{-1}\right)\right) & \text { if } s<t, \\ g\left(\frac{i}{2}\left(\varrho-\varrho^{-1}\right)\right) & \text { if } s>t .\end{cases}
$$

Proof. Using polar coordinates $z=\frac{1}{2}\left(u+u^{-1}\right) \in \mathcal{E}_{\varrho}, u=\varrho e^{i \theta}, \theta \in[0,2 \pi), \varrho>1$, there follows

$$
\begin{aligned}
& \left|z^{2}-s^{2}\right|^{2}=\frac{1}{16}\left|u^{2}+u^{-2}+2-4 s^{2}\right|^{2} \\
& (4.1)=\frac{1}{16}\left(\left(\varrho^{2}+\varrho^{-2}+2-4 s^{2}\right)^{2}-4\left(2-4 s^{2}\right)\left(\varrho^{2}+\varrho^{-2}\right) \sin ^{2} \theta-4 \sin ^{2} 2 \theta\right) \\
& (4.2)=\frac{1}{16}\left(\left(\varrho^{2}+\varrho^{-2}-2+4 s^{2}\right)^{2}+4\left(2-4 s^{2}\right)\left(\varrho^{2}+\varrho^{-2}\right) \cos ^{2} \theta-4 \sin ^{2} 2 \theta\right) .
\end{aligned}
$$

In the case $s<t,(4.1)$ together with $a_{2}(\varrho):=\frac{1}{2}\left(\varrho^{2}+\varrho^{-2}\right)$ shows that

$$
|g(z)|^{2}=\frac{\left(a_{2}(\varrho)+1-2 s^{2}\right)^{2}-4\left(1-2 s^{2}\right) a_{2}(\varrho) \sin ^{2} \theta-\sin ^{2} 2 \theta}{\left(a_{2}(\varrho)+1-2 t^{2}\right)^{2}-4\left(1-2 t^{2}\right) a_{2}(\varrho) \sin ^{2} \theta-\sin ^{2} 2 \theta} \leq\left|g\left(\frac{1}{2}\left(\varrho+\varrho^{-1}\right)\right)\right|^{2}
$$

for all $z \in \mathcal{E}_{\varrho}$ if and only if $\varphi_{1}(\theta) \leq 0, \theta \in[0,2 \pi)$, where

$$
\begin{aligned}
\varphi_{1}(\theta) & :=8 a_{2}(\varrho)\left(\left(1-2 s^{2}\right)\left(1-2 t^{2}\right)-a_{2}^{2}(\varrho)\right) \sin ^{2} \theta+4\left(a_{2}(\varrho)+1-s^{2}-t^{2}\right) \sin ^{2} 2 \theta \\
& =\left\{a_{2}(\varrho)\left(\left(1-2 s^{2}\right)\left(1-2 t^{2}\right)-a_{2}^{2}(\varrho)\right)+2\left(a_{2}(\varrho)+1-s^{2}-t^{2}\right) \cos ^{2} \theta\right\} \sin ^{2} \theta .
\end{aligned}
$$

In the case $s>t,(4.2)$ shows that

$$
|g(z)|^{2}=\frac{\left(a_{2}(\varrho)-1+2 s^{2}\right)^{2}+4\left(1-2 s^{2}\right) a_{2}(\varrho) \cos ^{2} \theta-\sin ^{2} 2 \theta}{\left(a_{2}(\varrho)-1+2 t^{2}\right)^{2}+4\left(1-2 t^{2}\right) a_{2}(\varrho) \cos ^{2} \theta-\sin ^{2} 2 \theta} \leq\left|g\left(\frac{i}{2}\left(\varrho-\varrho^{-1}\right)\right)\right|^{2}
$$

for all $z \in \mathcal{E}_{\varrho}$ if and only if $\varphi_{2}(\theta) \leq 0, \theta \in[0,2 \pi)$, where

$$
\begin{aligned}
\varphi_{2}(\theta) & :=8 a_{2}(\varrho)\left(\left(1-2 s^{2}\right)\left(1-2 t^{2}\right)-a_{2}^{2}(\varrho)\right) \cos ^{2} \theta+4\left(a_{2}(\varrho)-1+s^{2}+t^{2}\right) \sin ^{2} 2 \theta \\
& =\left\{a_{2}(\varrho)\left(\left(1-2 s^{2}\right)\left(1-2 t^{2}\right)-a_{2}^{2}(\varrho)\right)+2\left(a_{2}(\varrho)-1+s^{2}+t^{2}\right) \sin ^{2} \theta\right\} \cos ^{2} \theta .
\end{aligned}
$$

Obviously, $\varphi_{\nu}(\theta) \leq 0$ for $\theta \in[0,2 \pi), \nu=1,2$, if and only if

$$
d_{\nu}(\varrho):=a_{2}(\varrho)\left(\left(1-2 s^{2}\right)\left(1-2 t^{2}\right)-a_{2}^{2}(\varrho)\right)+2\left(a_{2}(\varrho)+(-1)^{\nu}\left(s^{2}+t^{2}-1\right)\right) \leq 0 .
$$

For $s, t \in(0,1)$ the conditions $\left(1-2 s^{2}\right)\left(1-2 t^{2}\right) \leq 1$ and $(-1)^{\nu}\left(s^{2}+t^{2}-1\right) \leq 1$, $\nu=1,2$, are valid. Hence,

$$
d_{\nu}(\varrho) \leq-a_{2}^{3}(\varrho)+3 a_{2}(\varrho)+2=-\left(a_{2}(\varrho)+1\right)^{2}\left(a_{2}(\varrho)-2\right) \leq 0
$$

if $a_{2}(\varrho)-2=\frac{1}{2}\left(\varrho^{2}+\varrho^{-2}\right)-2 \geq 0$, i.e., $\varrho \geq \frac{\sqrt{2}}{2}(1+\sqrt{3})=: \varrho^{*}$.

Consequently, $\varphi_{\nu}(\theta) \leq 0$ for all $\theta \in[0,2 \pi)$ and $\nu=1,2$, if $\varrho \geq \varrho^{*}$, which proves the assertion. 
Remark 4.1. The proof shows that $\varrho^{*}$ is the smallest parameter for which the statement in Lemma 4.1 holds for any choice of $s, t \in(0,1)$. In the case $s<t$ the limit $\varrho^{*}$ is attained for $s, t \rightarrow 0$, and in the case $s>t$ for $s, t \rightarrow 1$.

Lemma 4.2. Let $w$ and $\widetilde{w}$ be symmetric weight functions on $(-1,1)$ with $\widetilde{w}(x)>$ $0, x \in(0,1)$. Then the quotient $q_{n}:=\widetilde{\pi}_{n} / \pi_{n}$ of the corresponding nth-degree orthogonal polynomials $\pi_{n}$ and $\widetilde{\pi}_{n}$ has the property that on $\mathcal{E}_{\varrho}$ with $\varrho \geq \varrho^{*}:=$ $\frac{\sqrt{2}}{2}(1+\sqrt{3})$ there holds

(a) $\max _{z \in \mathcal{E}_{\varrho}}\left|q_{n}(z)\right|=q_{n}\left(\frac{1}{2}\left(\varrho+\varrho^{-1}\right)\right)$ if $w / \widetilde{w}$ is increasing on $(0,1)$,

(b) $\max _{z \in \mathcal{E}_{\varrho}}\left|q_{n}(z)\right|=q_{n}\left(\frac{i}{2}\left(\varrho-\varrho^{-1}\right)\right)$ if $w / \widetilde{w}$ is decreasing on $(0,1)$.

Proof. Using the notations of Lemma 2.1, we see from (2.1) that

$$
q_{n}(x)=\frac{\widetilde{\pi}_{n}(x)}{\pi_{n}(x)}=\frac{\widetilde{c}_{n}}{c_{n}} \prod_{k=1}^{\left[\frac{n}{2}\right]} \frac{x^{2}-\left(\widetilde{x}_{k}^{(n)}\right)^{2}}{x^{2}-\left(x_{k}^{(n)}\right)^{2}} .
$$

If $w / \widetilde{w}$ is increasing on $(0,1)$, Lemma 2.1 shows that $x_{k}^{(n)} \geq \widetilde{x}_{k}^{(n)}, k=1, \ldots,\left[\frac{n}{2}\right]$. Applying Lemma 4.1 to each factor yields Lemma 4.2a.

If $w / \widetilde{w}$ is decreasing on $(0,1)$, Lemma $4.2 \mathrm{~b}$ follows again from Lemma 4.1, since now the inequalities $x_{k}^{(n)} \leq \widetilde{x}_{k}^{(n)}, k=1, \ldots,\left[\frac{n}{2}\right]$, hold by Remark 2.1.

Lemma 4.3. For $n \geq 1$ we have on every ellipse $\mathcal{E}_{\varrho}$ with $\varrho \geq \widehat{\varrho}_{n}$ that

$$
\max _{z \in \mathcal{E}_{\varrho}}\left|\frac{z}{U_{n}(z) U_{n+2}(z)}\right|=\frac{\varrho-\varrho^{-1}}{2\left|U_{n}\left(\frac{i}{2}\left(\varrho-\varrho^{-1}\right)\right) U_{n+2}\left(\frac{i}{2}\left(\varrho-\varrho^{-1}\right)\right)\right|},
$$

where $\widehat{\varrho}_{n}:=1+\sqrt{2}$ if $n$ is odd, and if $n$ is even, $\widehat{\varrho}_{n}$ is the greatest zero of

$$
d_{n}(\varrho):=\left(\varrho-\varrho^{-1}\right)^{2}-4-\left(\varrho^{2}-\varrho^{-2}\right)^{2}\left(\frac{(n+1)^{2}}{\left(\varrho^{n+1}+\varrho^{-n-1}\right)^{2}}+\frac{(n+3)^{2}}{\left(\varrho^{n+3}+\varrho^{-n-3}\right)^{2}}\right) .
$$

For $n$ even, one has $\widehat{\varrho}_{2}>\widehat{\varrho}_{4}>\cdots>\widehat{\varrho}_{n}>\lim _{\substack{n \rightarrow \infty \\ n \text { even }}} \widehat{\varrho}_{n}=1+\sqrt{2}$.

Proof. Let $z=\frac{1}{2}\left(u+u^{-1}\right) \in \mathcal{E}_{\varrho}$ with $u=\varrho e^{i \theta}, \theta \in[0,2 \pi), \varrho>1$, and

$$
\psi(z):=\frac{z}{U_{n}(z) U_{n+2}(z)}=\frac{\left(u+u^{-1}\right)\left(u-u^{-1}\right)^{2}}{2\left(u^{n+1}-u^{-n-1}\right)\left(u^{n+3}-u^{-n-3}\right)} .
$$

Short calculations using elementary properties of the trigonometric functions yield for the numerator

$$
\begin{aligned}
& \left|\left(u+u^{-1}\right)\left(u-u^{-1}\right)^{2}\right|^{2}=\left|\left(u^{3}+u^{-3}\right)-\left(u+u^{-1}\right)\right|^{2} \\
& =\left(\varrho-\varrho^{-1}\right)^{2}\left(\varrho+\varrho^{-1}\right)^{4}-4\left(4 \sin ^{2} 2 \theta+\left(\varrho+\varrho^{-1}\right)^{2}\left(\left(\varrho-\varrho^{-1}\right)^{2}-4 \sin ^{2} \theta\right)\right) \cos ^{2} \theta \\
& =: h_{1}(\theta),
\end{aligned}
$$


and for the denominator

$$
\begin{aligned}
\mid & \left.\left(u^{n+1}-u^{-n-1}\right)\left(u^{n+3}-u^{-n-3}\right)\right|^{2} \\
= & \left|\left(u^{2 n+4}+u^{-2 n-4}\right)-\left(u^{2}+u^{-2}\right)\right|^{2} \\
= & \left(\left(\varrho^{2 n+4}+\varrho^{-2 n-4}\right) \cos (2 n+4) \theta-\left(\varrho^{2}+\varrho^{-2}\right) \cos 2 \theta\right)^{2} \\
& +\left(\left(\varrho^{2 n+4}-\varrho^{-2 n-4}\right) \sin (2 n+4) \theta-\left(\varrho^{2}-\varrho^{-2}\right) \sin 2 \theta\right)^{2} \\
= & \left(\varrho^{n+1}+(-1)^{n} \varrho^{-n-1}\right)^{2}\left(\varrho^{n+3}+(-1)^{n} \varrho^{-n-3}\right)^{2}+4\left(\cos (2 n+4) \theta+(-1)^{n} \cos 2 \theta\right)^{2} \\
& -2\left(\varrho^{n+3}+(-1)^{n} \varrho^{-n-3}\right)^{2}\left((-1)^{n}+\cos 2(n+1) \theta\right) \\
& -2\left(\varrho^{n+1}+(-1)^{n} \varrho^{-n-1}\right)^{2}\left((-1)^{n}+\cos 2(n+3) \theta\right) \\
= & : h_{2}(\theta) .
\end{aligned}
$$

Hence, the condition $|\psi(z)|^{2} \leq\left|\psi\left(\frac{i}{2}\left(\varrho-\varrho^{-1}\right)\right)\right|^{2}$ is valid for $z \in \mathcal{E}_{\varrho}$ if and only if $\varphi(\theta) \leq 0$ for $\theta \in[0,2 \pi)$, where

$$
\begin{aligned}
\varphi(\theta):= & h_{2}\left(\frac{\pi}{2}\right) h_{1}(\theta)-h_{1}\left(\frac{\pi}{2}\right) h_{2}(\theta) \\
=-4 h_{2}\left(\frac{\pi}{2}\right)\{ & \left.4 \sin ^{2} 2 \theta+\left(\varrho+\varrho^{-1}\right)^{2}\left(\left(\varrho-\varrho^{-1}\right)^{2}-4 \sin ^{2} \theta\right)\right\} \cos ^{2} \theta \\
-2 h_{1}\left(\frac{\pi}{2}\right)\{ & 2\left(\cos (2 n+4) \theta+(-1)^{n} \cos 2 \theta\right)^{2} \\
& -\left(\varrho^{n+3}+(-1)^{n} \varrho^{-n-3}\right)^{2}\left((-1)^{n}+\cos 2(n+1) \theta\right) \\
& \left.-\left(\varrho^{n+1}+(-1)^{n} \varrho^{-n-1}\right)^{2}\left((-1)^{n}+\cos 2(n+3) \theta\right)\right\} .
\end{aligned}
$$

For $n$ odd, the assertion immediately follows since

$$
\varphi(\theta) \leq-4 h_{2}\left(\frac{\pi}{2}\right)\left(\varrho+\varrho^{-1}\right)^{2}\left(\left(\varrho-\varrho^{-1}\right)^{2}-4\right) \cos ^{2} \theta \leq 0 \quad \text { if } \quad \varrho \geq \widehat{\varrho}_{n}:=1+\sqrt{2} .
$$

For $n$ even, one obtains from (4.3), using the quantities $a_{m}(\varrho)$ and the inequality $\cos ^{2}(2 m+1) \theta \leq(2 m+1)^{2} \cos ^{2} \theta$ (cf. [6, Lemma 3.1]),

$$
\begin{aligned}
\varphi(\theta) \leq & -256 a_{n+1}^{2}(\varrho) a_{n+3}^{2}(\varrho) a_{1}^{2}(\varrho)\left(\left(\varrho-\varrho^{-1}\right)^{2}-4\right) \cos ^{2} \theta \\
& +64 a_{1}^{2}(\varrho)\left(\varrho^{2}-\varrho^{-2}\right)^{2}\left(a_{n+3}^{2}(\varrho) \cos ^{2}(n+1) \theta+a_{n+1}^{2}(\varrho) \cos ^{2}(n+3) \theta\right) \\
\leq & -256 a_{n+1}^{2}(\varrho) a_{n+3}^{2}(\varrho) a_{1}^{2}(\varrho) d_{n}(\varrho) \cos ^{2} \theta,
\end{aligned}
$$

with $d_{n}$ as defined in the lemma.

Obviously, $d_{n}(\varrho)<0$ if $\varrho \leq 1+\sqrt{2}$. For $\varrho \geq 1+\sqrt{2}$, by Lemma $2.2 \mathrm{c}$, one obtains $d_{n}(\varrho)<d_{n+2}(\varrho)<\lim _{n \rightarrow \infty} d_{n}(\varrho)=\left(\varrho-\varrho^{-1}\right)^{2}-4$, and by Lemma $2.2 \mathrm{~d}$ that $d_{n}(\varrho)$ is a strictly increasing function of $\varrho$ (for fixed $n$ ). Hence, for each even $n \geq 2$, there exists a unique parameter $\widehat{\varrho}_{n}>1+\sqrt{2}$ with $d_{n}\left(\widehat{\varrho}_{n}\right)=0$; moreover, $\widehat{\varrho}_{n}>\widehat{\varrho}_{n+2}>\lim _{n \rightarrow \infty} \widehat{\varrho}_{n}=1+\sqrt{2}$. For $\varrho \geq \widehat{\varrho}_{n}$, this yields $d_{n}(\varrho) \geq 0$ and therefore $\varphi(\theta) \leq 0$ for $\theta \in[0,2 \pi)$.

Combining the results for $n$ even and $n$ odd yields the assertion.

Remark 4.2. Equation (4.3) shows that $\varphi\left(\frac{\pi}{2}\right)=\varphi^{\prime}\left(\frac{\pi}{2}\right)=0$ for all $\varrho>1$. Since $\varphi(\theta) \leq 0, \theta \in[0,2 \pi)$, the point $\theta=\frac{\pi}{2}$ must be a local maximum of $\varphi$ and therefore $\varphi^{\prime \prime}\left(\frac{\pi}{2}\right) \leq 0$. Differentiating $\varphi(\theta)$ twice yields from (4.3)

$$
\varphi^{\prime \prime}\left(\frac{\pi}{2}\right)=-8\left(\varrho^{n+1}+(-1)^{n} \varrho^{-n-1}\right)^{2}\left(\varrho^{n+3}+(-1)^{n} \varrho^{-n-3}\right)^{2}\left(\varrho+\varrho^{-1}\right)^{2} \widehat{d}_{n}(\varrho),
$$


where

$$
\begin{aligned}
\widehat{d}_{n}(\varrho) & :=\left(\varrho-\varrho^{-1}\right)^{2}-4 \\
& -(-1)^{n}\left(\varrho^{2}-\varrho^{-2}\right)^{2}\left(\frac{(n+1)^{2}}{\left(\varrho^{n+1}+(-1)^{n} \varrho^{-n-1}\right)^{2}}+\frac{(n+3)^{2}}{\left(\varrho^{n+3}+(-1)^{n} \varrho^{-n-3}\right)^{2}}\right) .
\end{aligned}
$$

For $n$ even, we have $d_{n}(\varrho)=\widehat{d}_{n}(\varrho)$ and the equation $d_{n}(\varrho)=0$, i.e., $\varphi^{\prime \prime}\left(\frac{\pi}{2}\right)=0$, characterizes the optimal parameter for the maximum relation to hold (i.e., $\widehat{\varrho}_{n}$ is optimal for even $n)$. For $n$ odd, there holds $\lim _{n \rightarrow \infty} \widehat{d}_{n}(\varrho)=\left(\varrho-\varrho^{-1}\right)^{2}-4$, i.e., the parameter $\widehat{\varrho}_{n}=1+\sqrt{2}$ is asymptotically optimal in the sense that for $n \rightarrow \infty$ Lemma 4.3 cannot hold for any smaller parameter.

Lemma 4.4. For $n \geq 2$ we have on every ellipse $\mathcal{E}_{\varrho}$ with $\varrho \geq \widetilde{\varrho}_{n}$ that

$$
\max _{z \in \mathcal{E}_{\varrho}}\left|\frac{z}{T_{n}(z) T_{n+2}(z)}\right|=\frac{\left(\varrho+\varrho^{-1}\right)}{2 T_{n}\left(\frac{1}{2}\left(\varrho+\varrho^{-1}\right)\right) T_{n+2}\left(\frac{1}{2}\left(\varrho+\varrho^{-1}\right)\right)},
$$

where the parameter $\widetilde{\varrho}_{n}$ is the greatest zero of

$$
\widetilde{d}_{n}(\varrho):=(n+2)^{2}\left(\frac{\varrho+\varrho^{-1}}{\varrho^{n+2}+\varrho^{-n-2}}\right)^{2}+n^{2}\left(\frac{\varrho+\varrho^{-1}}{\varrho^{n}+\varrho^{-n}}\right)^{2}-1 .
$$

In particular, $\widetilde{\varrho}_{2}=2.41388, \widetilde{\varrho}_{3}=2.00166, \widetilde{\varrho}_{n} \leq \frac{\sqrt{2}}{2}(1+\sqrt{3})$ for $n \geq 4$.

Proof. Again writing $z=\frac{1}{2}\left(u+u^{-1}\right), u=\varrho e^{i \theta}, \theta \in[0,2 \pi), \varrho>1$, yields

$$
\psi(z):=\frac{z}{T_{n}(z) T_{n+2}(z)}=\frac{2\left(u+u^{-1}\right)}{\left(u^{n}+u^{-n}\right)\left(u^{n+2}+u^{-n-2}\right)}
$$

and

$$
|\psi(z)|^{2}=\frac{a_{1}^{2}(\varrho)-\sin ^{2} \theta}{\left(a_{n}^{2}(\varrho)-\sin ^{2} n \theta\right)\left(a_{n+2}^{2}(\varrho)-\sin ^{2}(n+2) \theta\right)} .
$$

Hence, the condition $|\psi(z)|^{2} \leq\left(\psi\left(\frac{1}{2}\left(\varrho+\varrho^{-1}\right)\right)\right)^{2}$ is valid for $z \in \mathcal{E}_{\varrho}$ if and only if $\varphi(\theta) \leq 0$ for $\theta \in[0,2 \pi)$, where

$$
\begin{aligned}
\varphi(\theta):= & a_{n}^{2}(\varrho) \sin ^{2}(n+2) \theta+a_{n+2}^{2}(\varrho) \sin ^{2} n \theta \\
& -\frac{a_{n}^{2}(\varrho) a_{n+2}^{2}(\varrho)}{a_{1}^{2}(\varrho)} \sin ^{2} \theta-\sin ^{2}(n+2) \theta \sin ^{2} n \theta .
\end{aligned}
$$

Employing $\sin ^{2} m \theta \leq m^{2} \sin ^{2} \theta, m \in \mathbb{N}$ (cf. [6, Lemma 3.1]), yields

$\varphi(\theta) \leq-\frac{a_{n}^{2}(\varrho) a_{n+2}^{2}(\varrho)}{a_{1}^{2}(\varrho)} \widetilde{d}_{n}(\varrho) \sin ^{2} \theta \quad$ with $\quad \widetilde{d}_{n}(\varrho):=(n+2)^{2} \frac{a_{1}^{2}(\varrho)}{a_{n+2}^{2}(\varrho)}+n^{2} \frac{a_{1}^{2}(\varrho)}{a_{n}^{2}(\varrho)}-1$.

According to Lemma $2.2 \mathrm{~d}$, for each $n$ there exists a unique parameter $\widetilde{\varrho}_{n}$ with $\widetilde{d}_{n}\left(\widetilde{\varrho}_{n}\right)=0$. Since $\lim _{\varrho \rightarrow \infty} \widetilde{d}_{n}(\varrho)=-1$, this implies $\varphi(\theta) \leq 0, \theta \in[0,2 \pi)$, if $\varrho \geq \widetilde{\varrho}_{n}$ and the assertion follows. 
Applying Newton's method yields $\widetilde{\varrho}_{2}:=2.41388$ and $\widetilde{\varrho}_{3}=2.00166$. Because of $a_{n}(\varrho) \geq b_{n}(\varrho)$ and Lemma $2.2 \mathrm{~b}$ one obtains for $n \geq 4$ and $\varrho>\frac{\sqrt{2}}{2}(1+\sqrt{3})$

$$
\begin{aligned}
\widetilde{d}_{n}(\varrho) & \leq a_{1}^{2}(\varrho)\left(\frac{(n+2)^{2}}{b_{n+2}^{2}(\varrho)}+\frac{n^{2}}{b_{n}^{2}(\varrho)}\right)-1 \\
& \leq 32 \frac{a_{1}^{2}(\varrho)}{b_{4}^{2}(\varrho)}-1=\frac{32}{\left(\varrho-\varrho^{-1}\right)^{2}\left(\varrho^{2}+\varrho^{-2}\right)^{2}}-1<0,
\end{aligned}
$$

which shows $\widetilde{\varrho}_{n} \leq \frac{\sqrt{2}}{2}(1+\sqrt{3})$ for $n \geq 4$.

Remark 4.3. There holds $\varphi(0)=\varphi^{\prime}(0)=0$ for all $\varrho>1$. Since $\varphi(\theta) \leq 0$ for $\theta \in[0,2 \pi)$, the point $\theta=0$ must be a local maximum of $\varphi$ and therefore $\varphi^{\prime \prime}(0) \leq 0$. The equation $\widetilde{d}_{n}(\varrho)=0$, i.e., $\varphi^{\prime \prime}(0)=0$, characterizes the optimal parameter for which the maximum relation is valid (i.e., $\widetilde{\varrho}_{n}$ is optimal).

Proof of Theorem 3.2. According to Theorem 3.1, the kernel $K_{n}$ of the Gaussian quadrature rule satisfies

$$
K_{n}(z)=\sum_{\nu=0}^{\infty} \frac{c_{n+2 \nu+2}}{c_{n+2 \nu}} \psi_{\nu}(z) \quad \text { with } \quad \psi_{\nu}(z):=\frac{z}{\pi_{n+2 \nu+2}(z) \pi_{n+2 \nu}(z)}, \nu \geq 0,
$$

and by Lemma 3.1 it is sufficient to study $\max _{z \in \mathcal{E}_{e}}\left|\psi_{\nu}(z)\right|, \nu \geq 0$.

(a) Let $w(x) \sqrt{1-x^{2}}$ be increasing on $(0,1)$. In terms of the Chebyshev polynomials $T_{m}$ the functions $\psi_{\nu}$ for $n \geq 2, \nu \geq 0$ can be rewritten as

$$
\psi_{\nu}(z)=\frac{z}{T_{n+2 \nu}(z) T_{n+2 \nu+2}(z)} \frac{T_{n+2 \nu}(z)}{\pi_{n+2 \nu}(z)} \frac{T_{n+2 \nu+2}(z)}{\pi_{n+2 \nu+2}(z)} .
$$

Lemma $4.2 \mathrm{a}\left(\right.$ with $\left.\widetilde{w}(x):=\left(1-x^{2}\right)^{-1 / 2}\right)$ yields for $\varrho \geq \frac{\sqrt{2}}{2}(1+\sqrt{3})$

$$
\max _{z \in \mathcal{E}_{\varrho}}\left|\frac{T_{n+2 \nu}(z)}{\pi_{n+2 \nu}(z)} \frac{T_{n+2 \nu+2}(z)}{\pi_{n+2 \nu+2}(z)}\right|=\frac{T_{n+2 \nu}\left(\frac{1}{2}\left(\varrho+\varrho^{-1}\right)\right)}{\pi_{n+2 \nu}\left(\frac{1}{2}\left(\varrho+\varrho^{-1}\right)\right)} \frac{T_{n+2 \nu+2}\left(\frac{1}{2}\left(\varrho+\varrho^{-1}\right)\right)}{\pi_{n+2 \nu+2}\left(\frac{1}{2}\left(\varrho+\varrho^{-1}\right)\right)} ;
$$

moreover, Lemma 4.4 (with the parameter $\widetilde{\varrho}_{n+2 \nu}$ ) shows for $\varrho \geq \widetilde{\varrho}_{n+2 \nu}$ that

$$
\max _{z \in \mathcal{E}_{\varrho}}\left|\frac{z}{T_{n+2 \nu}(z) T_{n+2 \nu+2}(z)}\right|=\frac{\varrho+\varrho^{-1}}{2 T_{n+2 \nu}\left(\frac{1}{2}\left(\varrho+\varrho^{-1}\right)\right) T_{n+2 \nu+2}\left(\frac{1}{2}\left(\varrho+\varrho^{-1}\right)\right)} .
$$

These two relations together with (4.4) imply

$$
\max _{z \in \mathcal{E}_{\varrho}}\left|\psi_{\nu}(z)\right|=\psi_{\nu}\left(\frac{1}{2}\left(\varrho+\varrho^{-1}\right)\right) \quad \text { for } \varrho \geq \max \left\{\widetilde{\varrho}_{n+2 \nu}, \frac{\sqrt{2}}{2}(1+\sqrt{3})\right\} \text { and } \nu \geq 0 \text {. }
$$

Since $\widetilde{\varrho}_{n} \leq \frac{\sqrt{2}}{2}(1+\sqrt{3})<\widetilde{\varrho}_{3}<\widetilde{\varrho}_{2}, n \geq 4$ (cf. Lemma 4.4), we set $\varrho_{n}^{*}:=\widetilde{\varrho}_{n}$ for $n=2,3$ and $\varrho_{n}^{*}:=\frac{\sqrt{2}}{2}(1+\sqrt{3})$ for $n \geq 4$. Then each term $\psi_{\nu}, \nu \geq 0$, attains its maximum modulus at $\frac{1}{2}\left(\varrho+\varrho^{-1}\right)$ for all ellipses $\mathcal{E}_{\varrho}$ with $\varrho \geq \varrho_{n}^{*}$. Lemma 3.1 now delivers the assertion.

(b) Let $w(x) / \sqrt{1-x^{2}}$ be decreasing on $(0,1)$.

Similar to the first case, we use the Chebyshev polynomials of the second kind, $U_{m}$, and get for $n \geq 1, \nu \geq 0$,

$$
\psi_{\nu}(z)=\frac{z}{U_{n+2 \nu}(z) U_{n+2 \nu+2}(z)} \frac{U_{n+2 \nu}(z)}{\pi_{n+2 \nu}(z)} \frac{U_{n+2 \nu+2}(z)}{\pi_{n+2 \nu+2}(z)} .
$$


Lemma $4.2 \mathrm{~b}\left(\right.$ with $\left.\widetilde{w}(x):=\sqrt{1-x^{2}}\right)$ yields for $\varrho \geq \frac{\sqrt{2}}{2}(1+\sqrt{3})$

$$
\max _{z \in \mathcal{E}_{\varrho}}\left|\frac{U_{n+2 \nu}(z)}{\pi_{n+2 \nu}(z)} \frac{U_{n+2 \nu+2}(z)}{\pi_{n+2 \nu+2}(z)}\right|=\left|\frac{U_{n+2 \nu}\left(\frac{i}{2}\left(\varrho-\varrho^{-1}\right)\right)}{\pi_{n+2 \nu}\left(\frac{i}{2}\left(\varrho-\varrho^{-1}\right)\right)} \frac{U_{n+2 \nu+2}\left(\frac{i}{2}\left(\varrho-\varrho^{-1}\right)\right)}{\pi_{n+2 \nu+2}\left(\frac{i}{2}\left(\varrho-\varrho^{-1}\right)\right)}\right| ;
$$

moreover, Lemma 4.3 (with the parameter $\widehat{\varrho}_{n+2 \nu}$ ) shows for $\varrho \geq \widehat{\varrho}_{n+2 \nu}$ that

$$
\max _{z \in \mathcal{E}_{\varrho}}\left|\frac{z}{U_{n+2 \nu}(z) U_{n+2 \nu+2}(z)}\right|=\frac{\varrho-\varrho^{-1}}{2\left|U_{n+2 \nu}\left(\frac{i}{2}\left(\varrho-\varrho^{-1}\right)\right) U_{n+2 \nu+2}\left(\frac{i}{2}\left(\varrho-\varrho^{-1}\right)\right)\right|} .
$$

These two relations and (4.5) imply for $\varrho \geq \widehat{\varrho}_{n+2 \nu}>\frac{\sqrt{2}}{2}(1+\sqrt{3})$ and $\nu \geq 0$

$$
\max _{z \in \mathcal{E}_{\varrho}}\left|\psi_{\nu}(z)\right|=\left|\psi_{\nu}\left(\frac{i}{2}\left(\varrho-\varrho^{-1}\right)\right)\right|=\frac{\varrho-\varrho^{-1}}{2\left|\pi_{n+2 \nu}\left(\frac{i}{2}\left(\varrho-\varrho^{-1}\right)\right) \pi_{n+2 \nu+2}\left(\frac{i}{2}\left(\varrho-\varrho^{-1}\right)\right)\right|} .
$$

Since $\sup _{\nu \geq 0} \widehat{\varrho}_{n+2 \nu}=\widehat{\varrho}_{n}$ (cf. Lemma 4.3), each term $\psi_{\nu}, \nu \geq 0$, attains its maximum modulus at $\frac{i}{2}\left(\varrho-\varrho^{-1}\right)$ for all ellipses $\mathcal{E}_{\varrho}$ with $\varrho \geq \widehat{\varrho}_{n}$. Lemma 3.1 yields the assertion with $\varrho_{n}^{*}:=\widehat{\varrho}_{n}$.

\section{Applichtions}

Theorem 3.2 permits us to locate the maximum modulus of $K_{n}(z)$ on suitable ellipses $\mathcal{E}_{\varrho}$ with $\varrho \geq \varrho_{n}^{*}$ for a great variety of Gaussian quadrature rules. In this section some special weight functions satisfying one of the conditions of Theorem 3.2 are studied.

Example 5.1. Consider the Gegenbauer weight functions $w^{(\alpha)}(x):=\left(1-x^{2}\right)^{\alpha}$, $\alpha>-1$. Here $\sqrt{1-x^{2}} w^{(\alpha)}(x)=\left(1-x^{2}\right)^{\alpha+\frac{1}{2}}$ is increasing on $(0,1)$ if $-1<\alpha \leq-\frac{1}{2}$ and $w^{(\alpha)}(x) / \sqrt{1-x^{2}}=\left(1-x^{2}\right)^{\alpha-\frac{1}{2}}$ is decreasing on $(0,1)$ if $\alpha \geq \frac{1}{2}$. Thus, Theorem 3.2 is applicable for $\alpha \notin\left(-\frac{1}{2}, \frac{1}{2}\right)$.

Theorem 5.1. The kernel $K_{n}^{(\alpha)}$ of the Gauss-Gegenbauer quadrature rule with respect to $w^{(\alpha)}(x)=\left(1-x^{2}\right)^{\alpha}, \alpha \notin\left(-\frac{1}{2}, \frac{1}{2}\right)$, satisfies on every ellipse $\mathcal{E}_{\varrho}$ with $\varrho \geq \varrho_{n}^{*}$

$$
\max _{z \in \mathcal{E}_{\varrho}}\left|K_{n}^{(\alpha)}(z)\right|= \begin{cases}K_{n}^{(\alpha)}\left(\frac{1}{2}\left(\varrho+\varrho^{-1}\right)\right) & \text { if }-1<\alpha \leq-\frac{1}{2}, n \geq 2, \\ \left|K_{n}^{(\alpha)}\left(\frac{i}{2}\left(\varrho-\varrho^{-1}\right)\right)\right| & \text { if } \alpha \geq \frac{1}{2}, n \geq 1 .\end{cases}
$$

The parameter $\varrho_{n}^{*}$ is for $\alpha \in\left(-1,-\frac{1}{2}\right]$ the one of Theorem $3.2 \mathrm{a}$ and for $\alpha \geq \frac{1}{2}$ the one of Theorem $3.2 \mathrm{~b}$.

In a few special cases, for example the Gauss-Chebyshev quadrature rules of the first and second kind (cf. $[7,8]$ ) and more generally the case $\alpha=k-\frac{1}{2}, k \in \mathbb{N}$ (cf. [11]), a detailed analysis yields a smaller parameter $\varrho_{n}^{(\alpha)}<\varrho_{n}^{*}$ for which the maximum relation in Theorem 5.1 is still valid. This analysis uses very special features of the weight function and the corresponding orthonormal polynomials. In the remaining cases $\alpha \in\left(-\frac{1}{2}, \frac{1}{2}\right)$ the method presented is not applicable since the necessary inequalities between the zeros of the corresponding orthonormal polynomials and the zeros of the Chebyshev polynomials are not valid (cf. Lemma 2.1). 
Nevertheless, a different method can be applied in these cases. This approach together with the discussion of the special cases $\alpha=k-\frac{1}{2}, k \in \mathbb{N}$, will be presented in a forthcoming paper.

Example 5.2. Let $p_{m}$ be a polynomial of degree $m$, symmetric on $(-1,1)$ and positive on $[0,1]$ and consider the symmetric Bernstein-Szegö weight functions $w_{m}^{(\alpha)}(x):=\frac{\left(1-x^{2}\right)^{\alpha}}{p_{m}(x)}, \alpha= \pm \frac{1}{2}$ (cf. [12]). Theorem 3.2 can be applied if appropriate assumptions on $p_{m}$ are made. For example, $\sqrt{1-x^{2}} w_{m}^{(-1 / 2)}(x)=\frac{1}{p_{m}(x)}$ is increasing on $(0,1)$ if and only if $p_{m}(x)$ is decreasing on $(0,1)$, and $w_{m}^{(1 / 2)}(x) / \sqrt{1-x^{2}}=$ $\frac{1}{p_{m}(x)}$ is decreasing on $(0,1)$ if and only if $p_{m}(x)$ is increasing on $(0,1)$.

In the important case $m=2$, Theorem $3.2 \mathrm{~b}$ holds for $w_{2}^{(1 / 2)}(x)=\frac{\sqrt{1-x^{2}}}{x^{2}+d^{2}}$ with $d>0$, and Theorem 3.2a holds for $w_{2}^{(-1 / 2)}(x)=\frac{1}{\sqrt{1-x^{2}}\left(d^{2}-x^{2}\right)}$ with $d>1$.

We note, that there exist some explicit representations for the kernels $K_{n}^{(m, \alpha)}$ of the corresponding Gaussian quadrature rules (cf. [10]). It is as yet unresolved how these can be used to determine $\max _{z \in \mathcal{E}_{o}}\left|K_{n}^{(m, \alpha)}(z)\right|$ or to derive smaller parameters than those of Theorem 3.2.

Example 5.3. Consider further special weight functions:

(a) For $w^{(\alpha)}(x):=e^{-x^{2}}\left(1-x^{2}\right)^{\alpha}, \alpha \geq \frac{1}{2}$, Theorem $3.2 \mathrm{~b}$ is applicable since $\frac{w^{(\alpha)}(x)}{\sqrt{1-x^{2}}}=e^{-x^{2}}\left(1-x^{2}\right)^{\alpha-\frac{1}{2}}$ is decreasing on $(0,1)$.

(b) For $w^{(\alpha, \gamma)}(x):=|x|^{\gamma}\left(1-x^{2}\right)^{\alpha}, \alpha \in\left(-1,-\frac{1}{2}\right], \gamma>0$, Theorem 3.2a is applicable since $w^{(\alpha, \gamma)}(x) \sqrt{1-x^{2}}=|x|^{\gamma}\left(1-x^{2}\right)^{\alpha+\frac{1}{2}}$ is increasing on $(0,1)$.

(c) For $w^{(\alpha)}(x):=-\left(1-x^{2}\right)^{\alpha} \log \left(1-x^{2}\right), \alpha \in\left(-1,-\frac{1}{2}\right]$, Theorem 3.2a is applicable since $w^{(\alpha)}(x) \sqrt{1-x^{2}}=-\left(1-x^{2}\right)^{\alpha+\frac{1}{2}} \log \left(1-x^{2}\right)$ is increasing on $(0,1)$.

\section{ACKNOWLEDGMENT}

I would like to thank the referee for helpful comments concerning the representation of this paper and for drawing my attention to [9].

\section{REFERENCES}

1. H. Brass, Quadraturverfahren, Vandenhoeck \& Ruprecht, Göttingen, 1977. MR 56:1675

2. P.J. Davis and P. Rabinowitz, Methods of numerical integration (second edition), Academic Press Inc. (London), 1984. MR 86d:65004

3. G. Freud, Error estimates for Gauss-Jacobi quadrature formulae, Topics in numerical analysis (J.J.H. Miller, ed.), Academic Press Inc. (London), 1973, pp. 113-121. MR 49:6563

4. W. Gautschi, On the remainder term for analytic functions of Gauss-Lobatto and GaussRadau quadratures, Rocky Mountain. J. Math. 21 (1991), 209-226. Corrected in W. Gautschi, Rocky Mountain. J. Math. 21 (1991), 1143. MR 93a:41071a; MR 93a:41071b

5. __ Remainder estimates for analytic functions, Numerical Integration (T.O. Espelid, A. Genz, eds.), Kluwer Academic Publishers, 1992, pp. 133-145. MR 94e:41049

6. W. Gautschi and S. Li, The remainder term for analytic functions of Gauss-Radau and Gauss-Lobatto quadrature rules with multiple end points, J. Comp. Appl. Math. 33 (1990), 315-329. MR 92a:65078

7. W. Gautschi and R.S. Varga, Error bounds for Gaussian quadrature of analytic functions, SIAM J. Numer. Anal. 20 (1983), 1170-1186. MR 85j:65010 
8. W. Gautschi, E. Tychopoulos and R.S. Varga, A note on the contour integral representation of the remainder term for a Gauss-Chebyshev quadrature rule, SIAM J. Numer. Anal. 27 (1990), 219-224. MR 91d:65044

9. D.B. Hunter, Some error expansions for Gaussian quadrature, BIT 35 (1995), 64-82.

10. F. Peherstorfer, On the remainder of Gaussian quadrature formulas for Bernstein-Szegö weight functions, Math. Comp. 60 (1993), 317-325. MR 93d:65030

11. T. Schira, Ableitungsfreie Fehlerabschätzungen bei numerischer Integration holomorpher Funktionen, Ph.D. Dissertation, Universität Karlsruhe, 1994.

12. G. Szegö, Orthogonal polynomials (fourth edition), American Mathematical Society Colloquium Publications, vol. 23, Providence, RI, 1975. MR 51:8724

Institut für Praktische Mathematik, Universität Karlsruhe, D-76128 Karlsruhe, GERMANY

E-mail address: schira@math.uni-karlsruhe.de 